\title{
LOS CONCEPTOS DE GRAMÁTICA, DE ATRIBUTO Y DE SUSTANTIVO EN ANDRÉS BELLO
}

Francisco Abad

$U N E D$

A Ricardo Senabre y

Darío Villanueva

OBJETO Y MÉTODO DEL ANÁLISIS GRAMATICAL

En el «Prólogo» de su Gramática y en el capítulo inicial de «Nociones preliminares", don Andrés Bello expone el objeto de conocimiento y los principios - tanto científicos como prácticos- que tiene presentes en la labor gramatical que entonces emprende.

En el «Prólogo» se manifiesta acerca de qué debe hacer una gramática, que no es sino hacer presentes, poner en claro de una manera completa y exacta, las reglas de hablar a las que obedece el buen uso del idioma castellano: «Hay puntos en que me ha parecido -declara así su propósito-, que las práctica de la lengua castellana podían representarse de un modo más completo y exacto... Las reglas a que se conforma el buen uso... no [pueden] exponerse con exactitud y fidelidad sino analizando, desenvolviendo los principios verdaderos que lo dirigen" 1 .

Se trata pues de analizar el objeto considerado de una manera "completa y exacta», esto es, adecuada empíricamente y de la mayor exhaustividad posible. La teoría o descripción gramatical de una lengua ha de recoger sus caracteres propios, ha de responder a la conformación o configuración que tal lengua tiene, viéndola en su exactitud y no a partir

1 Andrés Bello, Gramática de la lengua castellana destinada al uso de los americanos, edición critica de R. Trujillo, M. Almeida, M. Gómez, M. J. Artiles y J. Dorta, Santa Cruz de Tenerife, Cabildo Insular, 1981, p. 123. 
de analogías forzadas con la estructura de otros idiomas; Bello muy particularmente pone en guardia ante una latinización de las doctrinas teóricas, pues histórica e inadecuadamente se han sacado del latín la nomenclatura - dice- y los cánones gramaticales de nuestro romance ${ }^{2}$.

En realidad el artículo de 1832 de don Andrés «Gramática castellana», constituía ya un alegato en contra de la latinización no debida de la teoría gramatical de otras lenguas distintas de la romana; el escrito encierra buena parte también de las principales afirmaciones que estarán años más tarde en el «Prólogo» de la Gramática. Bello manifiesta entonces cómo la gramática nacional ha de exponer y clasificar los hechos del idioma, que no son otros que «las reglas del habla», y cómo ha de procurarse una acertada dirección para los hábitos del niño buscando una enseñanza de la lengua en la que nada ha de resultar vago ni oscuro, con palabras que no comprenda: estamos en lá grámatica nacional, subraya el venezolano, ante el primer asunto que aparece a la inteligencia infantil, "el primer ensayo de sus facultades mentales, su primer curso práctico de raciocinio» ${ }^{3}$.

Pero ya decimos que sobre todo estas páginas de 1832 encierran un rechazo a cualquier extrapolación inadecuada de las descripciones gramaticales del latín al idioma castellano. No basta con aprender la lengua romana para saber también la gramática de la nacional - argumenta-, sino que hace falta además un aprendizaje particular del idioma materno: «El que haya aprendido el latín (escribe a la letra) mucho mejor de lo que generalmente se aprende entre nosotros, sabrá el latín, y además habrá formado una mediana idea de la estructura del lenguaje y de lo que se llama gramática general; pero no sabrá por eso la gramática del castellano, porque cada lengua tiene sus reglas peculiares, su índole propia, sus genialidades, por decirlo así» ${ }^{4}$.

Bello rechaza por tanto que la Academia traiga a la gramática castellana el esquema de la declinación latina, o que contrariamente unifique en una sola categorías distintas, si en latín se habían dado superpuestas; en definitiva pide un estudio «rigurosamente analítico" de la lengua propia.

Por supuesto nuestro autor, además de postular un análisis exacto o adecuado de cada idioma en su particularidad, desea que tal análisis resulte también lo más completo posible, que sea completo tanto por in-

2 lbld., p. 124.

3 Andrés Bello, «Gramática castellana», O.C., V, Caracas, Ministerio de Educación, 1951, pp. 173-184: p.177. En p. 175 ha dicho: «La atención que el gobierno y el público de esta ciudad prestan actualmente al interesante objeto de la educación literaria hace esperar que no parecerán inoportunas las observaciones sigujentes sobre el primero de los estudios juveniles, que es al mismo tiempo uno de los más necesarios y de los más abandonados. Hablamos del estudio de la lengua patriax.

Ibid., p. 176. 
tensión - diríamos-, como por extensión. No sólo han de buscarse de esta manera los rasgos caracterizadores y actuales de la lengua, sino que habrá de tratar de conocerse toda la casuística que responda a fenómenos distintos del código idiomático. Ya decimos que don Andrés Bello se propone al frente de su Gramática representar las prácticas de la lengua castellana, de un modo más completo y exacto de lo que lo habian hecho hasta entonces las explicaciones usuales.

Modernamente se han establecido las exigencias de una teoría lingüística en la no contradicción, la exhaustividad y la sencillez, o también en la adecuación explicativa que es capaz de evaluar gramáticas propuestas de modo alternativo ${ }^{5}$; de estas condiciones las primeras son - según nos parece- las de no contradicción y de exhaustividad, pues ellas no llevarán a percibir de manera adecuada y completa (en el límite) el objeto estudiado.

Chomsky, interesado por lo antropológico humano, va más allá de la lengua en lo que ella importa a un filologo, pero a quien tiene sólo una sensibilidad y preocupación filológicas, lo que más debe importarle son las condiciones de no contradicción y de exhaustividad de la teoría; desde luego la gramática más sencilla posible resulta preferida, pero esta sencillez no ha de lograrse a costa de la pérdida de contenido empírico, y por tanto a costa de la exhaustividad y las no contradicciones.

Lo primero resulta por supuesto la adecuación al objeto, a la lengua cuya gramática se trata de establecer: que se clasifiquen y expongan las reglas del habla - según decía Bello-, de un modo lo más exacto y completo que esté al alcance del estudioso; así la gramática no tendrá contradicciones y empezará a acercarse a una cierta exhaustividad. Complementariamente se pide también a la teoría su coherencia interior, que esté simplificada sin por ello simplificar la complejidad y totalidad cognoscible de los hechos. Bien decía ya Louis Hjelmslev que la exigencia de falta de contradicción tiene preferencia sobre la de exhaustividad, y esta última sobre la simplicidad ${ }^{6}$.

Pero se trata no sólo de haber dejado en claro un principio de empirismo metodológico, sino de delimitar el objeto formal y el objeto material propios de la Gramática. Para don Andrés ken el lenguaje lo convencional y arbitrario abraza mucho más de lo que comúnmente se piensa", y por ello «cada lengua tiene su teoría particular», teoría que ha de procurar la mayor racionalidad y exhaustividad; sin duda estamos ante un afán de exactitud y lógica solidario de la idea condillaciana de que la construcción de un lenguaje perfecto da lugar a una ciencia per-

${ }^{5}$ Para esto último vid. -como es sabido- Noam Chomsky, Aspectos de la teoria de la sintaxis, Madrid, Aguilar, 1970.

${ }_{6}$ L. Hjelmslev, Prolegómenos a una teoria del lenguaje, Madrid, Gredos, 1971, pp. 22-23. 
fecta, ya que la ciencia es un lenguaje bien hecho. Pero además - como decimos-, se trata de establecer el objeto de estudio.

La gramática ha de ocuparse, enuncia Bello, de «el valor preciso de las inflexiones y las combinaciones de las palabras», es decir, de las inflexiones que presentan las voces y de la estructura de las oraciones ${ }^{7}$; nos hallamos ante el objeto científico-formal propio de los estudios gramaticales, mientras que su objeto material lo constituye el uso bueno, el que está vigente por la costumbre uniforme de las gentes educadas. Don Andrés por tanto posee una idea a la vez científica y normativa de la gramática: el estudioso ha de aclarar «las prácticas como la lengua las presenta», el uso en cuanto uso, pero limitándose a los empleos estimativamente considerados del idioma, aquellos estandarizados en la costumbre de los hablantes con cultura. El venezolano reconoce que esta actitud normativa está ya en Salvá, cuya Gramática de la lengua castellama - de la que ha procurado sacar provecho- constituye «un libro que ninguno de los que aspiran a hablar y escribir correctamente nuestra lengua nativa debe dispensarse de leer y consultar a menudo" ${ }^{8}$.

En definitiva, Bello se propone en su Gramática ilustrar la lengua por la lengua misma, según su constitución y sus principios inmanentes de funcionamiento, y hacerlo del modo más adecuado y completo posible; además dentro de todas las elocuciones imaginables se atiene a las acostumbradas por la gente educada.

\section{LA UNIDAD DEL IDIOMA}

El interés de nuestro autor por la educación idiomática está ya muy presente en su artículo de 1832, en el que expresa cómo el cultivo de la lengua retarda sus alteraciones y por tanto la uniforma, de donde resulta el bien práctico de la inteligibilidad entre contemporáneos o respecto de momentos históricos pasados. Sin duda una mentalidad ilustrada que aspira a satisfacer los intereses y la felicidad humanos, le hace a don Andrés proclamar al poco de haber llegado a Chile: «A proporción de la fijeza y uniformidad que adquieren las lenguas, se diminuye una de las trabas más incómodas a que está sujeto el comercio entre los diferentes pueblos, y se facilita asimismo el comercio entre las diferentes edades, tan interesante para la cultura de la razón y para los goces del entendimiento y del gusto" ${ }^{9}$. Como decimos, es un hombre de la Ilustración quien se expresa mediante estas palabras.

\footnotetext{
7 Gramática..., pp. 126 y 127.

" Ibid., p. 126.

" ¿Gramática castellana». p. 175.
} 
Luego la Gramática contendrá un pasaje bien conocido, en el que el venezolano vuelve a proclamar: «Juzgo importante - dice- la conservación de la lengua de nuestros padres en su posible pureza, como un medio providencial de comunicación y un vínculo de fraternidad entre las varias naciones de origen español derramadas sobre los dos continentes» ${ }^{10}$. Pone en guardia entonces ante la avenida que sufre el idioma de neologismos de construcción: con buen sentido advierte $\mathrm{Be}$ llo que no importa tanto la constante identidad de elementos del código lingüístico cuanto «la regular uniformidad de las funciones que éstos ejercen», de la que deriva la vitalidad preservadora de la lengua. Hoy preocupa también por ejemplo la inútil multiplicación del léxico ${ }^{11}$, pero desde luego las percepciones de don Andrés resultaban bien sensatas.

El venezolano acaba estimando globalmente que el valor pedagógico de la educación lingüística, más una acción preservadora de la unidad del idioma, le han llevado a hacer la Gramática; respecto de lo segundo, proclamará a la letra: «Sea que yo exagerare o no el peligro, él ha sido el principal motivo que me ha inducido a componer esta obra... Mi ambición quedará satisfecha con que... contribuya a la mejora de un ramo de enseñanza que no es ciertamente el más lucido, pero es uno de los más necesarios»

\section{EL NOMBRE DE LA LENGUA}

La Gramática de la lengua castellana destinada al uso de los americanos se inicia por unas «Nociones preliminares» cuyos dos primeros párrafos recogen en realidad ideas ya aparecidas en el Prólogo, pero expresadas ahora para quien va a estudiar, para quien se dispone a leer un tratado gramatical. «La GRAMÁTICA de una lengua -define don Andrés - es el arte de hablarla correctamente, esto es, conforme al buen uso, que es el de la gente educada. Se prefiere este uso porque es el más uniforme en las varias provincias y pueblos que hablan una misma lengua» 13 .

${ }^{10}$ Gramática..., p. 129.

1 Cfr. por ejemplo Dámaso Alonso, «Para evitar la diversificación de nuestra lenguaw, Presente y futuro de la Lengua Española, Madrid, MCMLXIV, II, pp. 259-268.

12 Gramática..., pp. 130-131. A partir de las afirmaciones conjuntas que hace Bello en todo el «Prólogo», creemos que no puede sostenerse -como hace José Jesús Gómez Asencio- que la Gramática de don Andrés resulta «normativa, pero no explicitamente pedagógica" (Gramática y categorias verbales en la tradición española. 1771-1847, Salamanca, Universidad, 1981, p. 32); el propio texto está dividido en dos cursos, señalados cada uno con letra de cuerpo mayor o menor.

${ }_{13}$ Op. cit., p. 139. 
Nos encontramos - pues - ante un conjunto de reglas que llevan al buen uso, y que garantizan por tanto una cierta uniformidad e inteligibilidad del idioma. Las reglas a que se conforma el buen uso constituyen el objeto de análisis para el estudioso, y en cuanto «arte» resultan la habilidad o competencia de que ha de hacer empleo el hablante. Ideas como la de la educación idiomática y la de la uniformidad y unidad lingüistica, bien caracterizadoras del pensamiento de Bello, reaparecen ahora en estos párrafos iniciales de su Gramática.

Enseguida don Andrés tiene que referirse al nombre de la lengua; su solución ya está anticipada en el propio título de la obra, pero ahora aparece de nuevo de manera explícita: «Se llama lengua castellana - dice- (y con menos propiedad española) la que se habla en Castilla y que con las armas y las leyes de los castellanos pasó a la América, y es hoy el idioma común de los Estados hispanoamericanos» ${ }^{14}$.

La denominación de «castellano», con adjetivo sustantivado, la ha creido Manuel Alvar del siglo XIV, si. bien José Mondéjar corrige a su maestro estableciendo que castellano se encuentra como adjetivo especificativo de lenguaje con anterioridad a 1289 , y se convierte en sustantivo después ${ }^{15}$. Andando los siglos, y con referencia a nuestra centuria, Amado Alonso ha advertido cómo el nombre de castellano para la denominación de la lengua es el que más se usa en la América hispanohablante; los motivos son - decía literalmente Amado- «arcaísmo (sobre todo fuera de las grandes ciudades), academicismo y celo patriótico» ${ }^{16}$. En nuestros días algunos autores empiezan a testimoniar que se emplea también el término español en las repúblicas hispanoamericanàs ${ }^{17}$.

La denominación castellano ha pervivido - pues- por siglos, debido, decía Amado Alonso, a la fuerza de la inercia; Alvar opone al maestro navarro que no se trata exactamente de un arcaísmo: "Castellano no es arcaísmo (escribe), porque se ha utilizado siempre y se sigue utilizando en la propia España por escritores que no son de Castilla; lo que sí es verdad es que muchos españoles de otras regiones prefieren el neologismo español porque en él se hallan cobijados y en el otro [en la otra denominación] no. Se dirá que son razones afectivas, cierto, pero contra

14 Ibid.

15 M. Alvar, "Para la historia de "castellano"", Homenaje a Julio Caro Baroja, Madrid, CIS, 1978, pp. 71-82; J. Mondéjar, "Castellano* $y$ "Español». Dos nombres para una lengua, Granada, Don Quijote, 1981.

${ }_{16}$ A. Alonso, Castellano, español, idioma nacional. Historia espiritual de tres nombres, Buenos Aires, Losada, $1968^{4}$.

17 Alvar escribe en concreto: «Hoy, incluso en los sitios donde se prefirió tradicionalmente castellano (Colombia, por ejemplo), el neologismo español va ganando crédito...Allí castellano planteaba la duda de la modalidad lingüística que debiera enseñarse (¿castellano con zeta, con elle, con vosotros?) * (loc. cit., p. 81, y cfr. con posterioridad su Hombre, etnia, Estado, Madrid, Gredos, 1986). 
ellas no hay argumento; la razón está allí donde el hablante quiere encontrarse; unos se identifican con castellano, otros con español, otros con ambas designaciones de su lengua» 18 .

Las opciones de Amado Alonso y de Alvar no son incompatibles: estamos ante un nombre arcaico conservado por pura continuidad o inercia, y que los escritores pueden preferir por ser una denominación técnica y patrimonial.

\section{PROSODIA}

Dispuesto a empezar los capítulos de la gramática, Bello enumera las partes del arte de hablar, y dice así: «El bien hablar comprende la estructura material de las palabras, su derivación y composición, la concordancia o armonía que entre varias clases de ellas ha establecido el uso, y su régimen o dependencia mutua. La concordancia y el régimen forman la construcción o sintaxis» ${ }^{19}$.

De hecho se referirá pues a la Sintaxis y la Analogía, aunque su separación le parezca imposible dada la unicidad de contenido y expresión en el lenguaje ${ }^{20}$. Las páginas primeras dedicadas a «Estructura material de las palabras» enseñan «la recta pronunciación y acentuación» de la que se ocupaba la tradicional Prosodia.

En la composición material del habla, distingue Bello sonidos elementales o letras; también se da el nombre de letras a los sonidos denotados por los signos alfabéticos, dice. Las palabras se resuelven en un corto número de sonidos elementales o irresolubles en otros: son las vocales y las consonantes, que nuestro autor enumera y cree que son cinco y veinte respectivamente ${ }^{21}$. Bello trata de veinte sonidos porque distingue $b$ y $v$, grafemas de un mismo fonema.

Junto a las unidades fónicas más elementales, el maestro venezolano se ocupa también de la sílaba y la palabra. «Combinándose unos con otros - dice- los sonidos elementales forman palabras, bien que basta a veces un solo sonido, con tal que sea vocal, para formar palabra»; a

18 «Para la historia...», p. 76.

19 Gramática..., p. 140.

20 Una «Nota» que puso en la edición de 1847 al capítulo de *Nociones preliminares* decía: «He omitido la división de la Gramática en Analogía y Sintaxis, porque la separación es imposible. ¿Quién no ve por ejemplo, que si ha de darse idea de lo que significa la palabra declinación, es preciso dar a conocer lo que es complemento directo y lo que es dativo? ¿Quién no ve que el género supone el conocimiento de la concordancia?» (Ibid., p. 743).

21 lbid., p. 141, si bien el texto aparece con algunas erratas de impresión. 
su vez «llámanse sílabas los miembros o fracciones de cada palabra, separables 0 indivisibles» 22 .

El acento lo interpreta Bello en cuanto resultado de la cantidad y el tono: «El acento consiste (escribe) en una levísima prolongación de la vocal que se acentúa, acompañada de una ligera elevación del tono» ${ }^{23}$.

\section{SUJETO Y ATRIBUTO, SUSTANTIVO Y VERBO}

Una lengua es una regular uniformidad de funciones, mantiene don Andrés, y por ello dedica el primer capítulo de su Gramática (el segundo estrictamente, ya que antes va el relativo a prosodia), a una «clasificación de las palabras por sus varios oficios».

Sostiene Bello que atendiendo a los varios oficios de las palabras en el razonamiento podemos reducirlas a siete clases, «sustantivo, adjetivo, verbo, adverbio, preposición, conjunción, interjección» ${ }^{24}$. El idioma expresa el pensamiento y se corresponde con él, parece querer decirnos, y en ese manifestarse el pensamiento las palabras tienen una capacidad o lugar, una función. Las palabras poseen oficios que hacen articularse el decurso para que esté constituido, y para que tal articulación se corresponda con el pensamiento y lo exprese.

Cualquier lengua consiste intrínsecamente en una estructura gramatical o una uniformidad de funciones, $y$ así el conocimiento de ese entramado permite ordenar el caudal de las voces. La uniformidad de funciones sirve como medio, para el fin de construir decursos que estén en correspondencia con el razonamiento y lo manifiesten.

Bello, pues, acorde con su idea de la naturaleza funcional-formal del idioma, empieza la gramática estableciendo las clases de palabras, que él tiene en siete. En la nota complementaria I insiste en que cada clase de palabras debe distinguirse de las demás por sus funciones peculiares, por las funciones que tiene en la expresión del razonamiento. "La clasificación de las palabras enuncia- es propiamente una clasificación de oficios gramaticales» 25 .

Distingue don Andrés siete especies de voces, según decimos, aunque indica que al sustantivo y al adjetivo puede genéricamente considerárseles

22 Ibid., p. 143.

23 lbid., p. 147. Una síntesis de opiniones y caracterizaciones sobre fonemas y sonidos, sílaba y acento está en A. Quilis y J. A. Fernández, Curso de fonética y fonologia espariolas, Madrid, CSIC, $1985^{\prime \prime}$, y A. Quilis, Fonética acústica de la lengua española. Madrid, Gredos, 1981.

24 Gramatica..., p. 149.

25 Ibid., p. 744. 
como «nombres», dada la semejanza de sus accidentes y la frecuente metábasis de uno en otro; no obstante, poseen caracteres exclusivos de importancia.

Las palabras de acuerdo con su clase tienen un orden o rango respectivo que Bello establece de esta manera:

El sustantivo es la palabra dominante; todas las otras concurren a explicarlo y determinarlo.

El adjetivo y el verbo son signos de segundo orden; ambos modifican inmediatamente al sustantivo.

El adverbio es un signo de orden inferior; modifica modificaciones.

Los adjetivos, verbos y adverbios no bastan para todas las modificaciones, mediatas o inmediatas, del sustantivo; hay otro medio destinado al mismo fin, que es el complemento. El complemento significa una relación y presenta necesariamente el objeto en que ésta termina, llamado término; a veces solo, a veces precedido de una palabra a que ha dado la lengua el oficio peculiar de anunciarlo. Esta palabra es la preposición.

El complemento, por lo dicho, o consta de término solo (la más veces denotado por un sustantivo), o de preposición y término. Él es, además, o un signo de segundo orden, como el adjetivo, o un signo de orden inferior, como el adverbio.

La conjunción no tiene propiamente rango; es un vínculo entre elementos análogos; liga sustantivos con sustantivos, adjetivos con adjetivos, verbos con verbos, adverbios con adverbios, oraciones con oraciones.

La interjección, en fin, es como un verbo inconjugable, que envuelve el sujeto, y está siempre en la primera persona del presente de indicativo ${ }^{26}$.

Vemos, pues, al maestro chileno referirse a las categorías en que se dividen las palabras, a sus oficios gramaticales o funciones, $y$ al orden o rango al que pertenecen. En la expresión del razonamiento las palabras tienen funciones que son su oficio gramatical, y de acuerdo con la función que cumplan en cada decurso concurren al mismo según un rango; para Bello la lengua es un sistema de consistencias formal-funcionales mediante las cuales se puede construir un decurso. El idioma existe a modo de cuerpo $u$ organismo viviente, pues en él se da una identidad y conexión de funciones: la trama formal, lingüistica, gramatical (como quiera decirse), que sirve de medio para la expresión de la sustancia de pensamiento. "Oficios» de las palabras, «funciones», «categorías», «orden» o «rango» de las mismas, constituyen conceptos con los que nuestro autor remite a la esencia funcional por la que existe una lengua.

En su clasificación de las palabras Bello argumenta funcionalmente que es la manera adecuada de hacerlo conforme a la propia naturaleza de los códigos idiomáticos; así muestra cómo cualquier frase sencilla de

${ }^{26}$ lbid., pp. 744-745. 
sentido completo consta de dos partes diversas, la que significa una cosa y la que nos da a conocer lo que pensamos acerca de ella: «Llámase la primera - dice- SUJETO O SUPUESTO, y la segunda ATRIBUTO» ${ }^{27}$. Pues bien; el carácter peculiar del sustantivo está en que es apto para servir de sujeto, y el del verbo en su aptitud para atributo.

La relación atributiva existe en el verbo respecto de un sustantivo, y también en el adjetivo predicado; nos hallamos no obstante con dos hechos distintos de la lengua, y así diferencia Bello:

\begin{abstract}
El adjetivo predicado, constante en su referencia al sustantivo, puede hallarse en muy diversos lugares, ya construyéndose inmediatamente con el sustantivo (la oscura noche, el triste invierno), ya modificando al verbo (el día amaneció tempestuoso), ya designando el término de un complemento (se acreditan de valientes, tiene fama de hermosa, da en temerario). Yo miro, pues, al predicado como una función del adjetivo, cuando refiriéndose al sustantivo sin limitar su extensión enuncia una cualidad del objeto que éste significa. Por consiguiente hago diferencia entre predicado y atributo. El adjetivo predicado y el verbo modifican ambos a un sustantivo; pero el segundo lo hace precisamente designando la segunda parte de la proposición, el atributo; presidiendo en él a todas las otras palabras que lo componen, y tomando las formas peculiares que corresponden a la persona y número del sujeto, y a las ideas de tiempo y de modo que conviene indicar; caracteres de que no goza el adjetivo predicado. Podrán preferirse otros términos para distinguir las dos cosas que yo llamo predicado y atributo; pero la distinción entre ambas es un hecho incontestable de la lengua.
\end{abstract}

El concepto de atributo, pues, lo guarda nuestro autor muy específicamente para designar la segunda parte de las dos de que consta toda proposición.

El atributo varía de forma con el número y persona del sujeto, y asimismo para significar tiempo; estas son las especies de variaciones de que es susceptible la forma del atributo en la proposición. Y, como ya hemos visto, el verbo es la clase de palabras que denota el atributo; en las proposiciones una palabra —explica Bello-, indica la persona y número del sujeto más el tiempo del atributo: «esta palabra es la más esencial del atributo; es por excelencia el atributo mismo, porque todas las otras de que éste puede constar no hacen más que referirse a ella, explicando o particularizando su significado. Llamámosla verbo. El VERBO es, pues, una palabra que denota el atributo de la proposición, indicando juntamente el número y persona del sujeto y el tiempo del mismo atributo» 28.

27 Ibid., p. 149.

28 Ibid., p. 151. 
No cabe tratándose de definiciones de enumerar sólo especies de verbos según lo que ellos signifiquen, sino de encontrar los rasgos que definan al verbo en cuanto una clase de palabras; don Andrés lo subrayará explícitamente ante la manera de proceder de otros autores 29 .

El maestro venezolano entiende, pues, que el sustantivo y el verbo llevan en su forma de contenido la propiedad de sujeto o atributo de la proposición respectivamente, y de ahí su orden o rango gramatical; nos encontramos por tanto ante un criterio definidor semántico-sintáctico que ve el conjunto de funciones en que consiste la lengua como la forma del contenido gramatical, diriamos hoy.

Los verbos no los define Bello por su distinta forma del contenido léxico, sino por la forma del contenido gramatical que es común a todos ellos, forma que les lleva a su oficio de atributo. El contenido gramatical respectivo de las palabras es el que determina su rango en la estructura de la cadena hablada, y en conjunto se logra así la armazón formal-funcional idiomática o código comunicativo. «Las funciones semánticas -escribe nuestro compañero Guillermo Rojo- constituyen el paralelo de las sintácticas en el plano del contenido» ${ }^{30}$; nos parece no obstante que en general lo sintáctico debe interpretarse también como (forma en el) plano del contenido.

Que en cualquier frase sencilla podemos reconocer sujeto o supuesto y atributo que - unidos- forman la proposición, tal como decía Andrés Bello, lo enfoca hoy por ejemplo Bernard Pottier con afirmaciones como estas:

Toda comunicación transmite un propósito.

El proposito tiene una estructuración teórica binaria: soporte y aporte.

El soporte es el elemento voluntariamente puesto, y se le llamará entidad, dotada de una visión de independencia.

El aporte se pone en cuanto un comportamiento, en visión de dependencia.

El propósito es la relación entre una entidad y un comportamiento ${ }^{31}$.

${ }^{29}$ En la nota III escribe: «Verbo (dice uno de nuestros más respetables gramáticos) es la parte de la oración que significa los movimientos o acciones de los seres, la impresión que éstos causan en nuestros sentidos, y algunas veces el estado de estos mismos seres, o la relación abstracta entre dos ideas. Ésta a mi juicio no es una definición de verbo, sino una enumeración de las diferentes especies de verbos según su significado; porque una definición debe mostrarnos el carácter común de todos los verbos, y lo que distinga a todos y a cada uno de ellos de las demás clases de palabras; faltando esto, no hay definicion» (lbid., p. 749).

3o G. Rojo, Aspectos básicos de sintaxis funcional, Málaga, Ágora, 1983, p. 75.

31 B. Pottier, Lingǘstica general, Madrid, Gredos, 1977, pp. 37-48. 
En cuanto al concepto de «atributo», Alarcos propone que se entienda por tal al término adyacente a un núcleo verbal de conmutación bastante limitada (ser, estar, quedar,...), término que desde el punto de vista léxico constituye el centro del predicado: el café está amargo, el gato era molesto ${ }^{32}$. Con esos núcleos verbales «que constituyen un inventario muy escaso..., aparece un adyacente denominado atributo... Es como un elemento cuyo lexema 'llena', por así decirlo, la vaga referencia del lexema verbal» ${ }^{33}$.

\section{El SUSTANTIVo}

El verbo tiene como función exclusiva la de su oficio gramatical de atributo, mientras el sustantivo - expone Bello- ejerce funciones una de las cuales es la de «designar el sujeto de la proposición»; sustantivo es pues, la palabra esencial y primaria del sujeto, el cual puede también componerse de otras voces referidas todas ellas a ese sustantivo dominante, al que modifican ${ }^{34}$.

Don Andrés sugiere que en la proposición la palabra primaria y dominante es el sustantivo; una proposición se ha dicho que es un campo de fuerzas, pues sus componentes contraen entre sí relaciones estructurales, y Bello entiende que el centro de gravedad de tal campo de fuerzas sintáctico se halla en el sustantivo. «Como al verbo se refieren -escribe- todas las otras palabras del atributo, y al sustantivo todas las otras del sujeto, y como el verbo mismo se refiere a un sustantivo, ya se echa de ver que el sustantivo sujeto es en la proposición la palabra primaria y dominante, y a la que directa o indirectamente miran todas las otras de que la proposición se compone» ${ }^{35}$.

Que el sustantivo es dominante lo ha propuesto Chomsky en As. pectos, en tanto hipótesis que habrá de ser contrastada; apunta en efecto cómo verbo y adjetivo se seleccionan en términos de la libre elección del nombre sujeto u objeto, y afirma, enunciando explícitamente: «No hay alternativa en la selección de los Verbos en términos de los Nombres ( $y$, utilizando un argumento similar, los adjetivos en términos de los Nombres), y no viceversa... Si es así, es posible dar

32 Emilio Alarcos Llorach, Estudios de gramática funcional del español, Madrid, Gredos, $1980^{3}$, p. 159.

${ }^{33}$ Cfr. también para los fenómenos gramaticales en torno al xatributom el manual conjunto de E. Alarcos y sus discípulos Lengua Española, Madrid, Santillana, ed. de 1984, pp. 36-37.

\footnotetext{
34 Gramática..., pp. 151-152.

${ }^{35}$ Ibid., p. 152.
} 
otro paso significativo hacia la caracterización general de las categorías Nombre, Verbo, Adjetivo, etc. Entre las categorías léxicas, rotulamos Nombre la que es seleccionalmente dominante en el sentido de que su constelación de rasgos está determinada por una regla de subcategorización independiente del contexto, siendo trasladados sus rasgos a otras categorías léxicas mediante reglas seleccionales" ${ }^{36}$.

Siguiendo más el espíritu que la letra -con todo el tecnicismo subyacente a ellas - de estas palabras chomskyanas, pensamos por nuestra cuenta que ha de resultar bien ilustrativa la comprobación del rango del nombre en la estructura sintáctica u oracional.

Del número y la persona del sustantivo en tanto palabra dominante, expone Bello, depende la forma del verbo. En cuanto a su forma léxica de contenido, «los sustantivos - dice-significan directamente los objetos en que pensamos» ${ }^{37}$; «le substantif (son las palabras de Pottier) est particularisant: il ne désigne rien en dehors de son apport prédicatif» ${ }^{38}$.

36 Aspectos..., pp. 109 ss.

37 Gramatica.., p. 152.

38 B. Pottier, Introduction a l'étude linguistique de l'espagnol, Paris, Eds. Hispanoamericanas, 1972, p. 93. 